\title{
Safe gene vectors made simpler
}

\section{Manal A. Morsy and C. Thomas Caskey}

In December 1995, a panel of experts reporting to the US National Institutes of Health (Bethesda, MD) director Harold Varmus concluded that ". . .the great potential of gene therapy may be undermined by efforts to rush it into clinical trials prematurely, which in the long run could hinder progress rather than speed it up. .." Their recommendation was to go back to the drawing board to focus on developing better transfer vectors and animal models, and to improve understanding of disease pathophysiology ${ }^{1}$.

There is little doubt that adenoviral vectors are among the most efficient gene delivery vehicles currently in use. The host range is broad, they are efficiently taken up by nondividing cells in vivo, they do not integrate into chromosomal DNA (thus there is a reduced risk of insertional mutagenesis), and recently, they have been shown to be amenable to redirected tissue targeting ${ }^{2,3}$. Retroviruses, by contrast, integrate randomly, infect only dividing cells, and generally can be produced only at low titers $\left(10^{5}-10^{7} / \mathrm{ml}\right)$.

While adenoviruses are often the vector of choice, they have their own problemsmost notably, dual immunogenicity. Not only do they show leaky expression of viral proteins that render them stimulatory to cellular immune responses (which in turn rapidly eliminate infected cells in vivo ${ }^{4,5}$ ), but also, by virtue of their capsid proteins, they stimulate a humoral response that effectively neutralizes secondary administration of the vector in immunocompetent animals ${ }^{4}$.

The nature of the major immunogenic component of adenoviral vectors has generated much controversy of late: Is it the foreign transgene or is it the expressed viral proteins? Most likely, immunogenicity is due to many contributory factors, including the type of transgene used, the mouse strain or animal species tested, the purity of the viral preparation, and viral titer. Nonetheless, it is clear that leaky viral protein expression does take place in the current "replication-deficient adenoviral vectors" $"$. 6 . The time is ripe, therefore, for innovative, modified, safer

Manal A. Morsy is research fellow and C. Thomas Caskey is senior vice president at the department of human genetics, Merck \& Co., Inc., WP26A-3000, Sumneytown Pike, West Point,PA19486(manal_morsy@merck.com; caskey@merck.com). vehicles that are as efficient as adenoviral vectors, but lack the extra baggage.

In this issue, Fender et al.' have taken a creative step toward that goal. The mature adenovirus virion contains at least 11 structural proteins encapsulating the double-stranded DNA genome, the viral capsid consisting of 6 polypeptides, including 240 copies of the trimeric hexone (polypeptide II) and 12 copies of each pentameric pentone base (polypeptide II) and trimeric fiber. In their paper, Fender et al. succeed in generating an adenovirus dodecahedron (by in vitro assembly) using only the adenoviral pentone-a complex of pentone pentameric bases and trimeric fibers. In addition, they elegantly show the uptake and fate of the generated dodecahedrons in HeLa cells using immunofluorescence and electron microscopy.

To circumvent the problem of DNA delivery - the small size of the dodecahedron internal cavity precludes DNA packagingthe authors modified the trimeric fiber with a carboxyl terminus extension of 20 lysines, then attaching polylysine-tagged plasmid DNA. By testing gene delivery and expression in cell culture, they showed that DNA was liberated into the nucleus, resulting in gene expression.

The efficiency of cellular or in vivo uptake of the adenoviral dodecahedron compared to other gene delivery vehicles remains to be determined. It is also unclear whether this new vehicle can evade immune rejection in vivo, although the lack of hexones in these formulations may indeed reduce the impact of humoral responses to vector administration. Nonetheless, it will be important to determine its extent, if repeat administration is desirable, depending on the stability of transgene expression.

How does this new vehicle compare to the most recent generation of adenoviral vectors-referred to as the "helper-dependent" or "gutless" adenoviral vectors ${ }^{8}$ ? These recombinant viruses are synthesized virtually devoid of viral coding regions, so leaky viral protein expression is eliminated. They also have a carrying capacity of up to $40 \mathrm{~kb}$ of insert DNA, can be purified at high titers, and are as efficient as older generations of recombinant adenoviral vectors in infecting cells ${ }^{8,9}$.

At the end of the day, the superiority of one system over the other will be determined by several factors, including, of course, in vivo efficacy with regard to gene delivery and expression, and with respect to addressing the issues pertinent to the development of these modified vectors [i.e., immunogenicity (safety), persistence, and/or successful repeat administration]. If the efficiency of gene delivery is comparable in these different vectors, amenability to large-scale production and stability are likely to play compelling roles in determining success as practical, safe, and effective therapeutics.

1. Touchette, N. 1996. Nat. Med. 2:7-9

2. Morsy, M.A., Mitani, K., Clemens, P., Caskey, C.T. 1993. J. Am. Med. Assoc. 270:2338-2345.

3. Douglas, J.T et al. 1996. Nature Biotechnology 14:1574-1578.

4. Yifan Dai Y. et al. 1995. Proc. Natl. Acad. Sci. USA 92:1401-1405.

5. Yang, Y., Joose, K.U., Su, Q., Ertl, H.C.J., Wilson, J.M. 1996. Gene Ther. 3:137-144.

6. Tripathy, S.K., Black, H.B., Goldwasser, E., and Leiden J.M. 1996. Nat. Med. 2:545-550.

7. Fender, P., Ruigrok, R.W.H., Gout, E., Buffet, S., and Chroboczek, J. 1997. Nature Biotechnology 15:52-56.

8. Kochanek, S. et al. 1996.Proc, Natl. Acad. Sci. USA. 93:5731-5736.

9. Parks, R.J. etal. 1997. Proc. Natl. Acad. Sci. USA In press

\section{E. coli moves into the plastic age}

\section{Sang Yup Lee}

Over the past 25 years, petrochemicalderived plastics have become indispensable materials in modern life. These plastics,

Sang Yup Lee is professor at the department of chemical engineering and the BioProcess engineering research center, KAIST, 373-1 Kusong-dong, Yusong-gu, Taejon 305-701, Korea(leesy@sorak.kaist.ac.kr). however, are not biodegradable and cause significant waste disposal and contamination problems. Increasing awareness of the harmful effects of nonbiodegradable plastics on the environment, together with the growing body of legislation preventing their use in various consumer products, has created much interest in the development of biodegradable plastic materials.

One group of compounds of potential in 